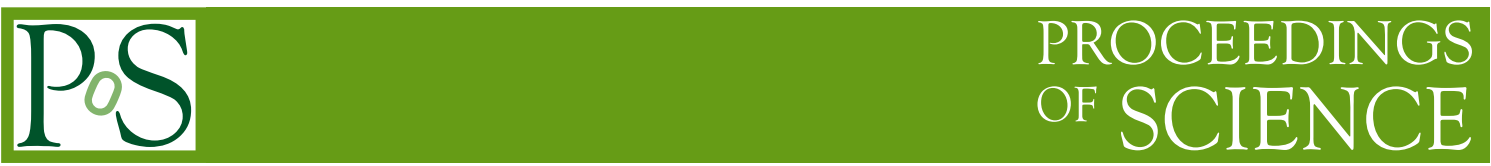

\title{
Recent highlights from the MAGIC telescopes
}

\section{Marcos López Moya ${ }^{* \dagger}$}

Univ. Complutense of Madrid

E-mail: marcos@gae.ucm.es

The MAGIC (Major Atmospheric Gamma-ray Imaging Cherenkov) telescopes were designed to reach the lowest possible energy threshold using the ground-based gamma-ray Cherenkov technique. In Fall 2009, the second telescope came into operation and MAGIC started stereo observations. During the last two years the performance of the instrument has been evaluated and new sources have been detected. The large mirror areas, along with the advanced technology used, allows MAGIC to reach an energy threshold of $50 \mathrm{GeV}$ in stereoscopic mode, being the integral sensitivity above $300 \mathrm{GeV}$ of $0.76 \% \mathrm{Crab}$ units (for $50 \mathrm{~h}$ integration). In this review, a selection of the most recent and conspicuous results obtained with MAGIC is exposed. For instance, we present the measurement of the Crab Nebula spectrum from $50 \mathrm{GeV}$ to $50 \mathrm{TeV}$; the detection of the Crab pulsar up to an energy of $400 \mathrm{GeV}$; the discovery at Very High Energies of two radiogalaxies (NGC 1275 and IC-310); the detection of numerous blazars and of the quasar PKS $1222+21$, and the measurement of the electron+positron spectrum between $100 \mathrm{GeV}$ and $3 \mathrm{TeV}$. MAGIC is currently undergoing a major upgrade of the trigger system and of the camera of the first telescope, to further improve its energy threshold and sensitivity.

The Extreme and Variable High Energy Sky - extremesky2011,

September 19-23, 2011

Chia Laguna (Cagliari), Italy

*Speaker.

${ }^{\dagger}$ On behalf of the MAGIC collaboration. 


\section{The MAGIC telescopes}

The two 17 meter diameter MAGIC telescopes constitute a last-generation instrument for Very High Energy (VHE) $\gamma$-ray observations, exploiting the Imaging Air Cherenkov (IAC) technique. Located on the Roque de los Muchachos Observatory, in La Palma island (Spain), MAGIC detects the faint flashes of Cherenkov light produced when $\gamma$-rays (or cosmic-rays) plunge into the earth atmosphere and initiate showers of secondary particles. The Cherenkov light emitted by the charged secondary particles is reflected by the mirrors of the telescopes and an image of the shower is obtained in each telescope camera. An offline analysis of the shower images allows the rejection of the hadronic cosmic ray background, the measurement of the direction of the incoming $\gamma$-rays, and the estimate of their energy. The first MAGIC telescope started observations in 2004, incorporating a number of technological improvements in its design, which allowed to achieve the lowest energy threshold (50 GeV with the nominal trigger, $25 \mathrm{GeV}$ with a special trigger for pulsar searches) among instruments of its kind, closing the gap between space-borne and ground-based instruments. The introduction of a second telescope in Fall 2009, enabled the instrument to perform stereoscopic observations with significantly better sensitivity. With respect to single telescope observations, a factor 2 to 3 improvement in significance is achieved. Above $300 \mathrm{GeV}$, the integral sensitivity is of $0.76 \%$ Crab units (for $50 \mathrm{~h}$ integration), with an angular and energy resolutions of $0.07^{\circ}$ and $\sim 16 \%$ respectively. For more details about the instrument's performance see [1].

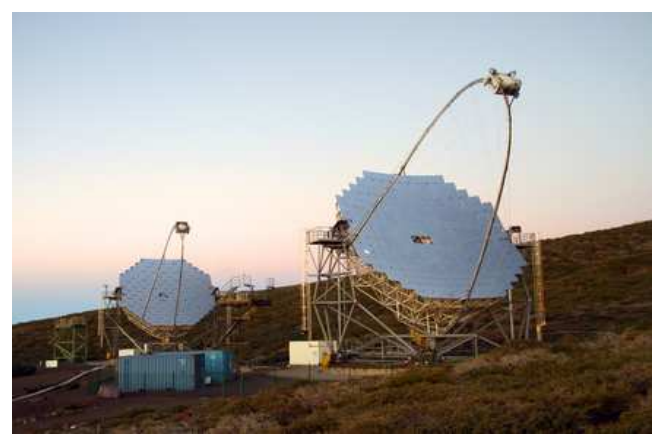

Figure 1: The two MAGIC telescopes at the Roque de los Muchachos observatory (La Palma Island, Spain).

\section{Galactic sources}

\subsection{The Crab nebula and pulsar}

The Crab Nebula is the standard candle for VHE astrophysics and an archetypal Pulsar Wind Nebula. At VHE the spectrum is dominated by Inverse Compton emission due to high energy electrons trapped by the nebular magnetic field. MAGIC has observed Crab in stereo mode for 49 hours during the past two winter seasons. The improved sensitivity of the instrument allows to produce a spectrum spanning three orders of magnitude in energy from $50 \mathrm{GeV}$ to $50 \mathrm{TeV}$ [2]. Figure 2 shows the combined fit of the Fermi/LAT and MAGIC data to a curved power law. The peak of the IC component of emission is found at $59 \pm 6 \mathrm{GeV}$.

A hot topic during the last year has been the discovery of fast variability in the Crab nebula at $\mathrm{GeV}$ energies [3]. MAGIC has searched for daily variations of the Crab Nebula flux over the 
above mentioned data sample, and also during a 3 hour observation period in April 2011 for which Fermi/LAT reports an increase of a factor 15 over the steady flux above $100 \mathrm{MeV}$. The flux measured by MAGIC was found to be steady within statistical errors during the whole analyzed data sample.

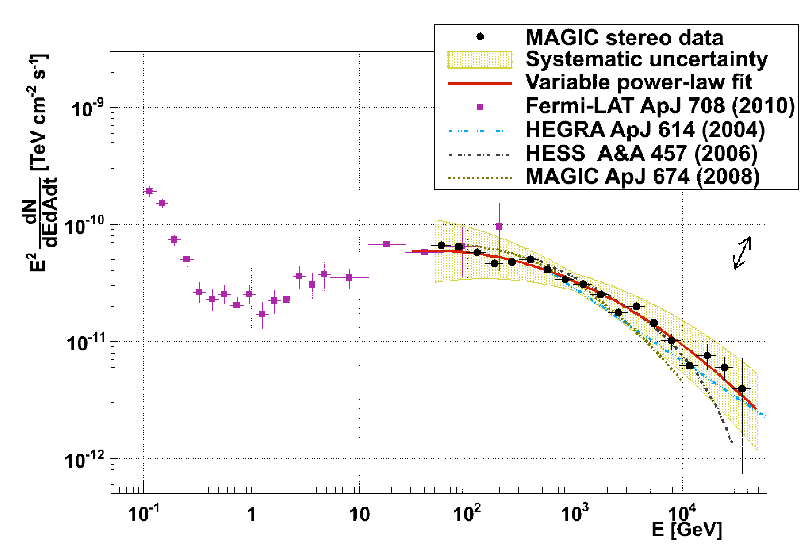

Figure 2: Spectral Energy Distribution of the Crab Nebula obtained by MAGIC during the past two winter seasons.

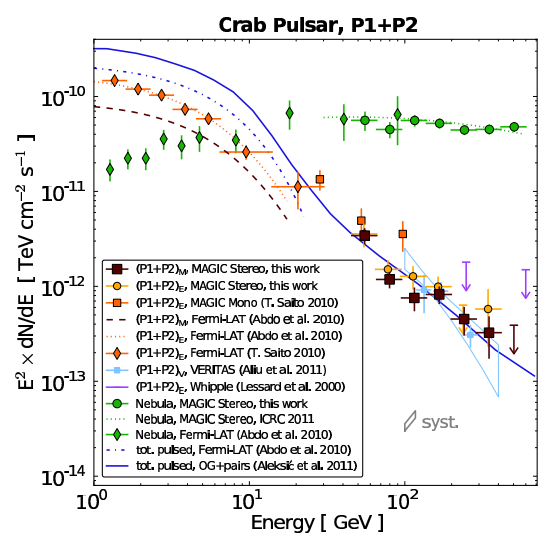

Figure 3: Crab pulsar spectrum obtained by MAGIC for the total pulsed emission.

The mechanism of the pulsed electromagnetic emission in the Crab pulsar is still an open fundamental question. Models for high energy emission (polar cap, outer or slot gap) predict exponential or superexponential cutoffs in pulsar spectra at a few $\mathrm{GeV}$, in agreement to what Fermi/LAT is measuring for all pulsars at $100 \mathrm{MeV}-10 \mathrm{GeV}$. MAGIC however detected pulsed emission from the Crab pulsar above $25 \mathrm{GeV}$, thanks to a special trigger system [4]. Recently, VERITAS also reported the detection of pulsed emission beyond $100 \mathrm{GeV}$ [7]. The analysis of 59 hours taken from Oct. 2007 to Feb. 2009 with MAGIC-I alone resulted in the first measurement of the Crab pulsar spectrum between $25 \mathrm{GeV}$ and $100 \mathrm{GeV}$, being even possible to obtain the spectra of the two peaks (P1 and P2) of the Crab pulsar light curve separately [5]. On the other hand, the analysis of 73 hours of data taking with the stereo system allowed to detect pulsed emission up to $400 \mathrm{GeV}$ [6]. The spectra of both $\mathrm{P} 1$ and $\mathrm{P} 2$ are compatible with power laws of photon indices $4.0 \pm 0.8$ (P1) and $3.42 \pm 0.26$ (P2), respectively. Figure 2 shows the combined spectrum of both peaks, for mono and stereo MAGIC data. The individual spectra are in good agreement within systematics and extend well beyond the spectral cutoff predicted by most of the polar cap or outer gap models.

\subsection{Extended sources}

The introduction of a second telescope improved the capabilities of MAGIC to study extended sources, as Pulsar Wind Nebula (PWN) and Supernova Remnants (SNR). Separate maps of the H.E.S.S. unidentified sources HESS J1857+026 and HESS J1923+141 could be produced at the energy bands $150-700 \mathrm{GeV}$ and above $700 \mathrm{GeV}$ [8]. The general morphology and spectral shape of the first source support a PWN origin for the $\gamma$-ray emission. The second source is in the field of view of the SNR W51C and has been detected by Fermi/LAT at GeV energies. The emission measured by MAGIC is spatially coincident with that reported by Fermi/LAT, but MAGIC's higher angular resolution shows that the bulk of the VHE $\gamma$-ray emission comes from the shocked molecular cloud located where the SNR shell collides with a large molecular cloud observed in radio data. 
This fact, and the better agreement of radio data with the hadronic scenario, suggests that the $\gamma$-ray emission is most likely of hadronic origin.

\subsection{Gamma-ray binaries}

After the discovery of LS I+61 303 in 2006, MAGIC has continued observing the source. The VHE emission decreased significantly in 2008, but, thanks to the increased sensitivity of the stereo system, MAGIC detected it at a flux of less than $5 \%$ of the Crab flux during this state of low emission in 2009 [9]. In Fall 2010 to Spring 2011 LS I+61 303 had returned to its previous level of VHE emission, in excess of $10 \%$ of Crab. This may point to a correlation with super-orbital variability due to modulation of the circumstellar wind.

Conversely, there is no evidence so far of VHE emission from microquasar jets. In this respect, MAGIC has recently completed a 4 year long multiwavelength campaign on Cyg X-3 [10], during which no VHE emission has been found. MAGIC monitored Cygnus X-1 for $50 \mathrm{~h}$ in 2006, and found an evidence of signal at the level of $4.1 \sigma$ on September 24th, 2006 [11]. The source was in a high state of emission and in coincidence with a hard X-ray flare. Following this promising result, MAGIC observed the source for an additional 90 hours between July 2007 and November 2009, however, no significant VHE signal was found in this sample.

\section{Extragalactic sources}

\subsection{Blazars}

Blazars constitute the vast majority of extragalactic sources detected at VHE. About 50 blazars have been so far detected in this energy range, 14 of them by MAGIC. Such impressive results are possible thanks to the low energy threshold of MAGIC and to the optical monitoring of selected target candidates with the KVA telescope (located also at La Palma island). This strategy resulted in the recent detection of the two blazars B3 2247+381 and 1ES 1215+303 [12]. Other recent results regarding this class of objects are: the detection of $3 \mathrm{C} 66 \mathrm{~A}$, whose redshift can be constrained to $\mathrm{z}<0.68$ from the spectra corrected for absorption by the Extragalactic Background Light (EBL); and the recent detection of $1 \mathrm{ES} 0647+250$, a BL Lac object of redshift $\mathrm{z} \sim 0.45$. In addition, during the past years, MAGIC has organized a good number of multiwavelength observations involving many instruments. In particular, since 2006 the nearby blazars Mrk 501 and Mrk 421 (see Fig. 4 [13]) are being monitored, to investigate potential spectral changes during periods of different source activity and search for potential periodic behavior.

\subsection{Flat Spectrum Radio Quasars (FSRQs)}

MAGIC has detected two out of the three quasars presently known to emit at VHE. 3C 279 $(\mathrm{z}=0.536)$ is the farthest VHE source detected so far. It was discovered by MAGIC at VHE in 2006 [14] and it allowed to place very strong constraint to the density of EBL. A second quasar, PKS $1222+21(4 \mathrm{C}+21.35, \mathrm{z}=0.432)$, has been detected by MAGIC in 2010 [15], during an increase of its $\mathrm{GeV}$ activity reported by Fermi/LAT. The VHE flux varied significantly within the 30 minutes of exposure implying a flux doubling time of about 10 minutes. The VHE and GeV spectra can be described by a single power law up to $400 \mathrm{GeV}$. The absence of a spectral cutoff at $30-60 \mathrm{GeV}$ 
constrains the $\gamma$-ray emission region to lie outside the broad line region, which would otherwise absorb the VHE $\gamma$-rays. Together with the detected fast variability, this fact challenges present emission models from jets in FSRQs.

\subsection{Radio galaxies}

MAGIC detected two out of the four known VHE radiogalaxies, namely NGC 1275 [16] and IC 310 [17], both in the Perseus cluster. NGC 1275 was detected during a period of enhanced GeV activity reported by Fermi/LAT. Its very steep spectrum made the detection possible thanks to the improved stereo sensitivity at $\mathrm{E}<100 \mathrm{GeV}$. IC 310 is the only radiogalaxy of the "head-tail" type detected so far.

\section{Other MAGIC physics topics}

\subsection{Measurement of the cosmic-ray electron spectrum}

Cherenkov telescopes may discriminate showers initiated by electrons from the background of hadronic CR showers through image shape analysis. A sample of 14 hours of observations has allowed to measure the combined $\mathrm{e}^{ \pm}$spectrum in the energy range between $100 \mathrm{GeV}$ and $3 \mathrm{TeV}$ [18], which is in good agreement with previous measurements, as seen in Fig. 5.

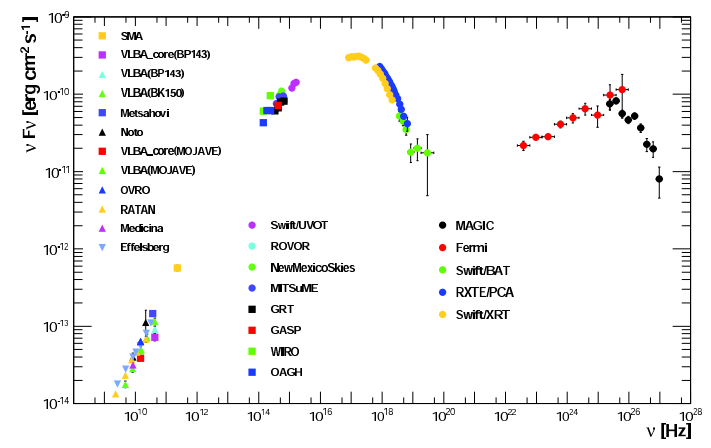

Figure 4: Spectral energy distribution of Mrk 421 averaged over all the observations taken during the multifrequency campaign in 2009.

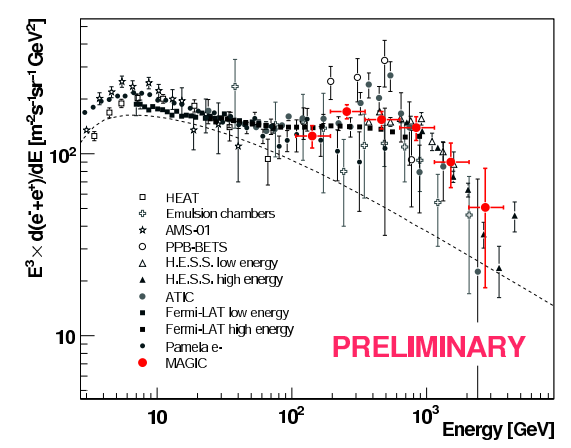

Figure 5: Spectrum of electrons and positrons measured with MAGIC, along with measurements from other instruments.

\subsection{Dark matter searches}

VHE $\gamma$-ray signatures of dark matter presence have been searched for with MAGIC in three dwarf satellite galaxies of the Milky Way with a high mass to luminosity ratio: Draco [19], Willman-1 [20] and Segue-1 [21], as well as in galaxy clusters as the Perseus cluster [22]. No emission has been detected and constraints on the physics or astrophysics parameters were derived. Limits are however still a factor 600 above the most optimistic expectations.

\section{Conclusions}

Based on a long Crab nebula observation the performance of the instrument has been evaluated, allowing to claim that MAGIC is right now the most sensitive observatory in the range $50-200$ 
$\mathrm{GeV}$. Highlights of the last two years of observations as detection of the Crab pulsar up to $400 \mathrm{GeV}$, or the discovery at VHE of a new quasar (PKS 1222+21) and two radiogalaxies (NGC 1275 and IC-310) were presented. The MAGIC telescopes were shut down in Summer 2011 to perform a major hardware intervention: both telescopes are now equipped with a new readout system, and the camera of MAGIC-I will be upgraded to match the geometry and trigger area of MAGIC-II. We expect an additional improvement in sensitivity, better performance for extended sources, and a further reduction of the energy threshold once the upgrade will be completed in Summer 2012.

\section{Acknowledgments}

The MAGIC collaboration thanks the Instituto de Astrofísica de Canarias for the excellent working conditions at the Observatorio del Roque de los Muchachos in La Palma. The support of the German BMBF and MPG, the Italian INFN, the Swiss National Fund SNF, and the Spanish MICINN is gratefully acknowledged. The author would also like to thank the support of the Ramón y Cajal program from the Spanish MICINN.

\section{References}

[1] J. Aleksić et al., accepted by Astrop. Phys (arXiv:1108.147)

[2] Zanin R. et al. for the MAGIC Coll., proc. 32nd ICRC, Beijing, 2011

[3] Tavani M. et al., Science $2011331: 736$

[4] Aliu E. et al., Science 2008 322:1221

[5] J. Aleksić et al., Ap. J. 2011 742:43

[6] J. Aleksić et al., accepted by A\&A (arXiv:1109.6124)

[7] Aliu et al., Science 2011 334: 69-72.

[8] Klepser, S. et al. for the MAGIC Coll., proc. 32nd ICRC, Beijing, 2011, and arXiv:1109.6448

[9] Jogler T., Blanch O. for the MAGIC Coll.,proc. 32nd ICRC, Beijing, 2011, and arXiv:1110.1681

[10] Aleksić J. et al., Ap. J. 2010 721:843

[11] Albert, J. et al., Ap. J. 2007 665:L51

[12] Colin P. et al. for the MAGIC Coll., proc. 32nd ICRC, Beijing, 2011, and arXiv:1110.0236

[13] Abdo A. A. et al., Ap. J. 2011 736:131

[14] Albert J. et al., Science 2008 320:1752

[15] Aleksić J. et al., Ap. J. Lett. 2011, 730:L8

[16] Hildebrand D. et al, for the MAGIC Coll. 2011 Proc. 32nd ICRC, Beijing, 2011

[17] Aleksić J et al. 2010 Ap. J. 723:L207

[18] Borla-Tridon D. et al. for the MAGIC Coll 2011 Proc. 32nd ICRC, Beijing, 2011

[19] Albert J. et al. Ap.J. 2008, 679:428

[20] Aliu, E. et al., Ap. J. 2009, 697:1229

[21] Aleksić J. et al., JCAP 2011 6:35

[22] Aleksić J. et al., A.\&A. 2010, 710:634 